\title{
La tutoría entre iguales como un potente recurso de aprendizaje entre alumnos: efectos, fluidez y comprensión lectora
}

\section{Peer tutoring as a powerful resource for learning among students: Effects on fluency and reading comprehension}

$\left(^{*}\right)$ Autor para correspondencia: Dra.Vanessa Valdebenito Zambrano

Profesor- Investigador, Facultad de Educación, Universidad Católica de Temuco. Manuel Montt 56, Of. 421, Campus San Francisco, Temuco, Chile. Correo electrónico: vvaldebenito@uct.cl

RECIBIDO:

30 de noviembre de 2012

ACEPTADO:

2 de abril de 2013

\author{
Dra. Vanessa Valdebenito Zambrano $\left({ }^{*}\right)$ \\ Facultad de Educación, Universidad Católica de Temuco, \\ Chile. \\ Dr. David Duran Gisbert \\ Universitat Autònoma de Barcelona, Cataluña. \\ España.
}

\section{Resumen}

El objetivo del estudio es investigar el impacto de las redes de cooperación en el aula, mediante un programa fundamentado en la tutoría entre iguales para promover el mejoramiento de la comprensión y fluidez lectora. En el programa Leemos en Pareja participan127 alumnos de $2^{\circ}$ a $5^{\circ}$ de primaria en 4 centros educativos de España. Con métodos mixtos de estudio, combinando un estudio cuasi-experimental con grupo de comparación (120 alumnos) e interpretándose los hallazgos por medio de un análisis cualitativo, se responde a los objetivos de la investigación. Los resultados muestran los avances obtenidos por los alumnos en comprensión y fluidez lectora, identificándose como elementos facilitadores del aprendizaje, la mediación ajustada y permanente que realizan los alumnos que asumieron el rol de tutores. Además de valorarse como una metodología que responde a la diversidad del alumnado, la tutoría entre iguales potencia el valor intrínseco de la cooperación.

Palabras clave: Aprendizaje cooperativo, tutoría entre iguales, aprendizaje entre profesores, competencia lectora, fluidez lectora.

\section{Abstract}

The aim of the study is to investigate the impact of cooperation networks in the classroom through an educational programme based on peer tutoring, to promote improvement in reading comprehension and fluency. 127 students in grades from second to fifth year of 4 schools in Spain participated in the programme "Leemos en Pareja" (Reading in Pairs). Mixed methods of study, combining a quasi-experimental design with comparison group (120 students) and interpreting the findings via a qualitative analysis, is used to respond to the research objectives. The results showed the progress made by the students in reading comprehension and fluency, identifying as facilitators of learning, mediation and permanent set performing students tutored by their peer tutors. Aside from being a valued methodology that responds to student diversity, peer tutoring enhances the intrinsic value of cooperation.

Keywords: Cooperative learning, peer tutoring, learning learning, reading comprehension, reading fluency. 


\section{INTRODUCCIÓN}

En la actualidad, los centros educativos buscan nuevas formas de potenciar el aprendizaje de sus alumnos/as, ajustándose a las características individuales y socioculturales de estos; entregando respuestas desde el seno de la comunidad educativa que movilizan a todos sus protagonistas a través de prácticas cada vez más inclusivas.

En este sentido, el aprendizaje cooperativo se ha situado en los últimos años como una importante estrategia en diversos contextos educativos con exitosos resultados, tanto en el ámbito del aula en los diferentes sub-sectores de aprendizaje (Vidal, Duran y Vilar, 2010; Mayoral y Corcelles, 2008; Moreno, 2007), como también respecto al trabajo en red que realizan los docentes (Fernández 2007; Monereo, Castelló, Bassols y Miquel, 2000; Utset y Duran -en prensa-). El trabajo fundamentado en la cooperación, permite asumir un compromiso individual y colectivo por medio de establecimiento de objetivos compartidos y consensuados que permiten a los participantes constituirse como un equipo para alcanzar las metas establecidas.

Además, la cooperación en sí misma es una competencia clave para la sociedad democrática del conocimiento (Rychen y Sagalnik, 2003) y de acuerdo a los planteamientos de la Comisión para la Educación del Siglo XXI (Delors et al., 1996), aprender a trabajar en equipo es una de las competencias básicas que deben facilitar los sistemas educativos. Es así también, como el aprendizaje cooperativo ha mostrado su eficacia frente a estructuras de trabajo competitivas e individualistas puestas en marcha en el aula, lo cual se explica y fundamenta en la teoría de la interdependencia social y sus variables moduladoras (interdependencia positiva; responsabilidad individual y grupal; interacción cara a cara; habilidades sociales; auto-reflexión de grupo), que median y aseguran la efectividad de la interacción (Johnson y Johnson, 2009). Como apunta Díaz-Aguado (2004), las investigaciones realizadas sobre la temática han permitido comprobar su contribución en las relaciones entre pares, profesorado, motivación por el aprendizaje y también el alcance de logros más allá del área curricular.

Uno de los métodos específicos del aprendizaje cooperativo, que ha reportado resultados satisfactorios en una gran diversidad de contextos educativos, es la tutoría entre iguales (peer tutoring). Con una importante tradición en el ámbito 
anglosajón y actualmente configurándose con exitosas experiencias en el contexto iberoamericano (Blanch, Duran, Valdebenito y Flores, 2012; Duran y Sánchez, 2012; Mosca y Santiviago, 2012; Duran y Huerta, 2008), la tutoría entre iguales es definida por Topping (2005) como la adquisición de conocimientos y habilidades a través de la ayuda activa y apoyo de pares o compañeros.

La tutoría entre iguales se fundamenta en la creación de parejas de personas que asumen una relación asimétrica, derivada de la adopción de un rol de tutor (enseñante) y/o tutorado (aprendiz), que trabajan por un objetivo común, conocido y compartido, que se logra gracias a un marco de interacción regulado por el docente (Duran y Vidal, 2004). En función de las características de la parejas, encontramos tutorías de distinta edad y, de la misma, en estas últimas de rol fijo o recíproco (Topping, 1988). Dicho método de aprendizaje cooperativo se sitúa en el marco conceptual del paradigma emergente, "aprender enseñando" -learning by teaching- (Duran, 2011; Cortese, 2005), que considera que la actividad de enseñar tiene un alto potencial de aprendizaje cuando se conforma a través de formatos bidireccionales de interacción. En este sentido, se entiende que el "tutor" aprende enseñando a su compañero tutorado, porque ello implica una preparación previa de los contenidos, actividades a desarrollar y la co-construcción de conocimientos gracias a la mediación, divergencia de opiniones e ideas, y consenso de respuestas. Por su parte, el "tutorado" aprenderá gracias a la mediación del compañero tutor, la ayuda personalizada y ajustada recibida, que le permitirá el avance desde su nivel de desarrollo real al nivel de desarrollo potencial.

Existen muchas investigaciones que han reportado los beneficios de diversas experiencias de tutoría entre iguales en la totalidad de etapas educativas y para distintas áreas curriculares. Por ejemplo, en comprensión lectora (Topping y Hogan, 1999; McMaster, Fuchs y Fuchs, 2006; Duran, Blanch, Thurston y Topping, 2010; Topping, Miller, Thurston, McGavock y Conlin, 2011); autoimagen lectora (Moliner, Flores y Duran, 2011); fluidez y velocidad lectora (Hattie, 2006; Valdebenito y Duran, 2010); escritura (Duran, Blanch, Dekhinet, Topping, 2010); matemáticas (Cohen, Kulik y Kulik 1982; Rohrbeck, Ginsburg-Block, Fantuzzo y Miller, 2003); ciencias (Thurston et al., 2007; Okilwa y Shelby, 2010); y competencia social (Byrd, 1990; Ginsburg-Block, Rohrbeck y Fantuzzo, 2006; Borisov y Reid, 2010). Además, muchas experiencias se han desarrollado también en contextos de multiculturalidad, vulnerabilidad social o educación especial (Robinson, Schofield y Streers-Wentzell, 2005; Van Keer y Verhaeghe, 2005; Spencer, 2006). 
Una de las áreas que destaca en los estudios revisados, debido a su carácter vehicular en el currículo escolar y como herramienta de aprendizaje a lo largo de toda la vida, es la lectura. Su constructo la enmarca dentro de la competencia lingüística y específicamente se vincula a la competencia lectora, siendo definida por la Organización para la Cooperación y el Desarrollo Económicos [OCDE] (2009) como la comprensión y utilización de textos escritos, y la reflexión e implicación para alcanzar los objetivos propios, desarrollar conocimiento y el potencial individual, para participar en la sociedad. De esta manera, la competencia lectora es concebida como un atributo indispensable para que los ciudadanos puedan desenvolverse en el medio social, constituyéndose como una pieza clave de enriquecimiento intelectual, adquisición de aprendizajes y acceso a la cultura (Gil, 2011). Así, tal y como señala la OCDE, un elemento esencial y distintivo del constructo de competencia lectora es la "comprensión lectora". Dicha comprensión se alcanzará producto de la relación que establezca el lector entre sus ideas y los atributos del texto, en un diálogo bidireccional, que permitirá la construcción de una representación que rescata elementos de ambas partes (Oakhill y Cain, 2007; Pearson y Hamm, 2005).

Como apuntan Nichols, Rupley y Rasinski (2009), el objetivo final de la lectura es la comprensión y el aprendizaje que se pueda alcanzar a partir de un texto, sin embargo el componente esencial que nutre y trae consigo dicha capacidad, radica en la fluidez lectora. Entendemos que podremos leer más y correctamente si entendemos lo que leemos, pero al mismo tiempo necesitamos leer fluidamente y de manera correcta para comprender. Garzón, Jiménez y Seda (2008) aclaran que la fluidez lectora es un constructo multidimensional entendido como la lectura realizada con velocidad, exactitud, características prosódicas apropiadas y la comprensión e interpretación del texto. 


\section{EL PROGRAMA LEEMOS EN PAREJA}

Tomando en cuenta los elementos reseñados anteriormente, se plantea el programa educativo Leemos en Pareja (Duran et al., 2009), en centros educativos de educación primaria de la Comunidad Autónoma de Aragón, España. Dicha propuesta pretende: poner al alcance de los profesores nuevas metodologías de trabajo con una clara orientación inclusiva; desarrollar nuevas didácticas para la enseñanza y aprendizaje de la lengua; generar y mantener redes de cooperación entre los docentes; fomentar la capacidad de cooperación entre los alumnos; mejorar la competencia lectora, especialmente la comprensión lectora del alumnado; y, potenciar la implicación de las familias en los trabajos escolares. Todos estos objetivos se fundamentan en tres pilares en que reposa el programa: la tutoría entre iguales, la comprensión lectora y la implicación familiar.

El programa pone en práctica una serie de actividades que involucran la lectura en pareja y comprensión lectora (entre alumnos en la escuela y entre familiar-hijo/a en el hogar), en formatos altamente estructurados, permitiendo de manera progresiva un uso más autónomo, ajustado y creativo, una vez que las parejas se van familiarizando con la dinámica y materiales de trabajo.

La primera acción contempla la formación inicial de los docentes que pondrán en marcha el programa en sus escuelas. Además, a través de un aula virtual los profesores comparten y construyen el material de lectura -hojas de actividades- que será el eje fundamental de las sesiones y que modularán la interacción de las parejas. De esta manera, se establece una red de cooperación entre los profesores de los diferentes centros ( $y$ también intra-centro), generándose interdependencia positiva para alcanzar un objetivo compartido, que en este caso será construir un dossier de hojas de actividades para las 48 sesiones de trabajo (24 sesiones en el aula y 24 en el hogar), compartiendo además inquietudes y reflexiones sobre el programa.

El paso siguiente se concreta en emparejar a los alumnos, entregarles la formación en relación al método tutoría entre iguales y los roles a asumir, todo ello bajo la responsabilidad del profesorado que guía el desarrollo de la actividad. Posteriormente, a lo largo de 12 semanas, a razón de dos sesiones semanales de 30 minutos, se pone en marcha el programa de lectura con los alumnos/as. 
Las actividades que contemplan las hojas de lectura-hojas de actividades-, promueven una anticipación a la lectura y establecimiento de hipótesis. Consecutivamente, se realizan tres lecturas en voz alta (lectura del tutor; lectura conjunta entre tutor y tutorado -Topping, 1987-; y, lectura del tutorado con la técnica pausa, pista y ponderación (PPP) -Wheldall y Colmar, 1990-, que permiten ir afianzando la construcción de la comprensión y fluidez lectora de los alumnos. En seguida se plantean una serie de actividades que promueven sobre todo la comprensión inferencial y de reflexión profunda a partir del texto. Finalmente, se contempla una lectura final en voz alta por parte del tutorado, que se espera realice con un ritmo, fluidez, velocidad y entonación adecuados propios de un conocimiento profundo del texto. Los alumnos tutores, avanzado el programa, acaban diseñando hojas de actividades para sus compañeros, respondiendo a sus intereses y requerimientos.

Paralelamente, la red de docentes continúa con encuentros presenciales y a través de una aula virtual, para valorar las estrategias implementadas, adaptar las hojas de actividades a las necesidades emergentes y compartir las experiencias a lo largo del desarrollo del programa. Finalmente, se cierra el programa con una valoración global de la implementación de la experiencia. 


\section{OBJETIVOS DE LA INVESTIGACIÓN}

1. Conocer el impacto de las redes de cooperación implementadas entre alumnos/as y entre profesores para mejorar la comprensión y fluidez lectora de los destinatarios del programa con la cuantificación de estos índices.

2. Determinar los efectos de la tutoría entre iguales respecto al rol asumido por los alumnos (tutor o tutorado) y el tipo de tutoría desarrollada (fija o recíproca), en relación a las variables de comprensión y fluidez lectora.

3. Analizar las percepciones que poseen los alumnos/as y profesores acerca de la metodología de trabajo, tutoría entre iguales.

\section{Método}

El trabajo se fundamenta en métodos mixtos de investigación, los cuales se enmarcan en el tercer paradigma de investigación educativa, que combina por parte de los investigadores el uso de métodos, técnicas y acercamientos cualitativos y cuantitativos en un solo estudio (Johnson y Onwuegbuzie, 2004).

Así, por una parte, el estudio involucra un enfoque cuasi-experimental, con una evaluación de tipo pre-test y post-test con grupo de comparación no equivalente. Las variables independientes corresponden a la tutoría entre iguales implementada por medio del programa Leemos en Pareja, y las variables dependientes son entendidas como la comprensión lectora, fluidez lectora, modalidad de tutoría y roles asumidos por los alumnos (tutor o tutorado).

Por otra parte, el estudio cualitativo a la luz de la información recuperada de cuestionarios y entrevistas realizadas, favorecería la comprensión de los posibles hallazgos en las variables objeto de estudio, interpretando qué y cuáles factores pudieran haber influido.

Como destacan Strijbos y Fischer (2007), a menudo, en las investigaciones fundamentadas en aprendizaje cooperativo (y colaborativo) son aplicadas metodologías de estudio mixtas, sin que ello pueda ir en detrimento del estudio mismo 
o como una incompatibilidad entre los instrumentos y procedimientos de recogida de datos, más bien y en concordancia con Jannsen, Kischner, Erkens, Kischner y Pass (2010) y Flick (2004) contribuiría a enriquecer los hallazgos del trabajo realizado.

Participantes

Grupo de intervención: Conformado por 127 alumnos/as, los cuales pertenecían a 4 centros educativos públicos de la provincia de Zaragoza y cursaban entre $2^{\circ}$ a $5^{\circ}$ de primaria: centro $A\left(4^{\circ}=28\right.$ alumnos; y, $\left.5^{\circ}=20\right)$; $B\left(4^{\circ}=22\right) ; C\left(3^{\circ}=19\right) ; y, D\left(2^{\circ}=38\right)$. Las edades de los participantes del grupo de intervención fluctuaban entre los 7 y 11 años. Los estudiantes de los centros $A, B$ y $D$ realizaron tutorías fijas, mientras que el grupo del centro $\mathrm{C}$ desarrolló tutoría recíproca entre sus miembros.

Grupo de comparación: Compuesto por 120 alumnos/as (69 niños y 51 niñas) de entre $2^{\circ}$ a $5^{\circ}$ de primaria de los mismos centros del grupo de intervención, pero que ni sus maestros ni ellos mismos participaban del programa.

Profesores: 8 en total que en el aula asumían el rol de profesor jefe o especialistas de la asignatura de lenguaje y comunicación.

\section{Instrumentos}

- Comprensión Lectora: Prueba ACL 10- $6^{\circ}$ Grado/ Evaluación de la comprensión lectora (Català, Comes y Renom, 2001). Compuestas por 710 textos, con 24- 36 ítems, con una fiabilidad KR-20 de .83 ACL 20; KR-20 de .80 ACL $3^{\circ}$; KR-20 de .83 ACL $4^{\circ}$; KR-20 de .82 ACL $5^{\circ}$.

- $\quad$ Fluidez Lectora: Se utilizó como parámetro de medición el componente velocidad lectora, para poder cuantificar el constructo de fluidez.

- $\quad$ Batería Psicopedagógica EVALUA 4 (García Vidal y González, 1999) y EVALUA 5 (García Vidal, Manjón y Ortiz, 2006), para alumnos/as de $4^{\circ}$ y $5^{\circ}$ de primaria. La prueba estandarizada posee una fiabilidad de .96 para la batería 4 y 0.845 para la 5 .

- $\quad$ Para $2^{\circ}$ y $3^{\circ}$ de primaria se tomó como referencia la prueba de velocidad lectora creada por el Ministerio de Educación de Chile [MINEDUC] (Plan de Mejoramiento Lector, s/f). 
- Cuestionario de valoración final del programa Leemos en Pareja (Duran et al., 2011), aplicado a los 127 alumnos/as participantes del estudio.

- Entrevistas semi-estructuradas a tutores y tutorados: Aplicadas a 16 estudiantes, al finalizar la experiencia, incorporando interrogantes sobre la satisfacción con el programa, ejercicio del rol, ayudas recibidas o entregadas, dificultades y progresos observados.

- $\quad$ Entrevistas a los docentes participantes: Realizadas a los 8 profesores participantes con el objetivo de profundizar en la organización e implementación del programa, valoración de la metodología, avances de los alumnos/as, y ventajas e inconvenientes del proceso.

\section{Análisis de los Datos}

Los datos cuantitativos fueron analizados a través software SPSS Stadistics 20, con la prueba t-student para muestras relacionadas y no paramétrica $\mathrm{W}$ de Wilcoxon.

Por otro lado, las respuestas de las entrevistas y cuestionarios aplicados, fueron analizados con el software atlas-ti 6.0, organizando los datos de acuerdo a un sistema de categoría de análisis, en base a los tópicos de las interrogantes, como: ayudas recibidas del tutorado; ayudas entregadas por el tutor, aprendizajes alcanzados por el tutor -tutorado; se alcanzan aprendizajes enseñando. En relación a las entrevistas de los profesores, se establecieron como categorías: avances de los alumnos con la metodología; identificación de factores que contribuyeron al aprendizaje. 


\section{RESULTADOS}

Los resultados del presente estudio son presentados en función de los objetivos de investigación.

1. Impacto de las redes de cooperación (entre alumnos y profesores) en los índices de comprensión y fluidez lectora de los alumnos/as participantes.

Comprensión Lectora: En dicho ámbito (Tabla 1), los resultados indican que el grupo de comparación no evidencia diferencias estadísticamente significativas entre pre y post-test, $t(119)=-1.81, p>.05$, reportando además un tamaño del efecto pequeño $(d=.25)$.

Sin embargo, los participantes del grupo de intervención, tras participar en el programa, alcanzaron avances estadísticamente significativos, $t(126)=-11.60, p<$ .01 , con un tamaño del efecto mediano $(d=.59)$. Se atribuyen los avances a las redes de cooperación implementadas a través de Leemos en Pareja.

\section{TABLA 1}

Resultados pre-test y post-test para grupo de intervención y grupo de comparación en relación a la comprensión y fluidez lectora

\begin{tabular}{|l|l|l|l|l|l|l|l|l|l|}
\hline Variable & Grupo & $\mathbf{N}$ & M Pre-test & SD & M Post-test & SD & $\mathbf{t}$ & $\mathbf{p}$ & $\mathbf{d}$ \\
\hline CL & GI & 127 & 56.14 & 18.54 & 67.09 & 18.41 & -11.60 & .00 & .59 \\
\hline \multirow{2}{*}{ FL } & GC & 120 & 51.42 & 18.29 & 55.94 & 18.54 & -1.81 & .07 & .25 \\
& GI & 127 & 57.64 & 19.35 & 73.87 & 20.41 & -12.35 & .00 & .82 \\
\hline & GC & 120 & 46.53 & 19.51 & 58.14 & 19.00 & -7.90 & .00 & .60 \\
\hline
\end{tabular}

Comprensión Lectora (CL); Fluidez Lectora (FL); Fuente: Elaboración propia; Grupo de Intervención (GI); Grupo de comparación (GC). Fuente: Elaboración propia.

Fluidez Lectora: La misma tabla (tabla 1), recoge que ambos grupos (grupo de comparación: $t(119)=-7.90, p<.01$; y, de intervención: $t(126)=-12.35$, $p<.01)$ muestran diferencias significativas entre las mediciones pre-test $y$ post-test. Se atribuyen los avances a que en ambos casos, los destinatarios han sido partícipes de actividades cotidianas que favorecían la práctica de la lectura en el aula. 
Para profundizar en la magnitud de las diferencias evidenciadas, se ha calculado el tamaño del efecto para ambos grupos. Los resultados en este sentido nos indican que los progresos obtenidos por el grupo de comparación se traducen en un tamaño del efecto mediano $(d=.60)$ y para el grupo de intervención se evidencia un tamaño del efecto grande $(d=.82)$. Estas diferencias en el tamaño del efecto, favorables para el grupo de intervención, son atribuidas a la práctica de la lectura durante todas las actividades implementadas comúnmente en el aula, pero sumada a las intervenciones puestas en marcha durante las sesiones de lectura del programa Leemos en Pareja y las redes de cooperación entre alumnos y profesores, las cuales han tenido un impacto profundo en los logros alcanzados en dicho componente de la competencia lectora.

Factores Explicativos: Los testimonios de los tutores $(\mathrm{T})$ nos ayudan a comprender la mediación realizada, la cual habría repercutido en el mejoramiento evidenciado, comentando que:

"no me resultaba difícil resolver las preguntas de comprensión lectora con el tutorado. Cuando él no las entendía le daba pistas, lo hacía pensar con otras cosas y al final, le acababa saliendo" (T2); "al tutorado a veces le costaba resolver las preguntas, así que yo tenía que indicarle cómo resolverlas" (T4); "a mi tutorada no le resultaba complicado, porque yo le indicaba donde podía encontrar la respuesta" (T6).

También, al ser consultados los tutores sobre sus progresos en fluidez lectora, el $90 \%$ de los tutores consideraba que leían mejor al finalizar el programa.

Por su parte, los tutorados (t) percibían en un $100 \%$ que habían mejorado su fluidez lectora, apuntando que:

"practicas y al final acabas mejorando" (t2); "creo que he mejorado porque cuando repetía la lectura me salía mucho mejor" (t8); "porque de tanto leer el texto ya sabía lo que venía” (t10).

La visión de los profesores $(\mathrm{P})$ respecto a estos avances también fue positiva y consecuente con los hallazgos, como se evidencia a continuación en los comentarios: "para la competencia lectora sí que ha servido, primero porque los niños no estaban acostumbrados a trabajar las preguntas de inferencia, nunca lo habíamos hecho, es la primera vez, por lo cual en las hojas hemos puesto y trabajado muchas preguntas de inferencia" (P1); "en algunos casos sí que han mejorado una barbaridad y han sacado provecho de la lectura, y en 
otros hemos conseguido más motivación que nada, justamente porque están más desmotivados por las tareas escolares y que hayan estado motivados por esta actividad ya es un avance" (P4); "los alumnos tutorados recibieron ayuda personalizada de un compañero más competente en lectura. Además sus compañeros tutores llegan con la ventaja enorme de haber realizado la ficha de lectura con anticipación en casa, con sus padres, por lo que les transmiten seguridad y también les transmiten la confianza y la cercanía de un igual, de un compañero. En este clima que se crea favorece un ambiente propicio para mejorar la competencia lectora. Por otra parte, también mejoran la competencia social y ciudadana, aprenden a aceptar ayuda y se relacionan más, ya que es una tarea cooperativa" (P6).

Los profesores además de apuntar que se evidencian progresos en la competencia lectora en general, señalan algunos factores que explicarían el éxito alcanzado, como: el trabajo a través de preguntas de contenido inferencial o reflexión profunda de las hojas de actividades; mayor motivación e involucramiento de los alumnos/as con bajas expectativas y desmotivados; mediación de un par que trasmite confianza y seguridad, favorece el clima de aprendizaje en el aula; y, el valor de la cooperación como una competencia fundamental para el desarrollo de la competencia social y ciudadana, esencial en la formación de los alumnos/as como miembros íntegros de una comunidad y la sociedad en general.

\section{Efectos de la tutoría entre iguales según la modalidad y rol, en relación a la fluidez y comprensión}

Para proceder con la máxima rigurosidad en relación a la obtención de los resultados, se ha realizado el análisis con la prueba t-student para muestras relacionadas para el grupo de alumnos/as de tutoría fija (que superaban los 30 sujetos); y se aplicó un análisis no paramétrico con la prueba $\mathrm{W}$ de Wilcoxon para el grupo de tutoría recíproca, ya que sus participantes no superaban 30 . Para este caso no se calculará el tamaño del efecto, ya que solo puede aplicarse a medidas paramétricas que poseen una distribución normal. Los resultados obtenidos en relación a la modalidad de tutoría asumida, se muestran en la tabla 2. 


\section{TABLA 2}

Resultados de las variables comprensión y fluidez lectora en función del tipo de tutoría fija o recíproca asumida.

\begin{tabular}{|l|l|l|l|l|l|l|l|l|}
\hline Variable & Tipo de Tutoría & $\mathbf{N}$ & $\mathbf{M}$ Pre-test & $\mathbf{S D}$ & $\mathbf{M}$ Post-test & $\mathbf{S D}$ & $\mathbf{t} / \mathbf{z}$ & $\mathbf{p}$ \\
\hline CL & Fija & 105 & 54.67 & 19.02 & 65.19 & 19.09 & -10.19 & .000 \\
\hline \multirow{2}{*}{ FL } & Recíproca & 22 & 63.15 & 14.43 & 76.13 & 11.15 & -3.77 & .000 \\
\hline & Fija & 105 & 60.45 & 19.58 & 78.44 & 18.73 & -12.86 & .000 \\
\hline
\end{tabular}

En relación a la comprensión lectora, se evidencian avances estadísticamente significativos en la tutoría fija, $\mathrm{t}(104)=-10.19, \mathrm{p}<.01$ para todos los participantes. En el caso de la tutoría recíproca, los resultados también son alentadores y muestran diferencias significativas entre las medidas iniciales y finales, $z=-3.77 ; p<.01$.

Respecto a la fluidez lectora, los resultados muestran diferencias estadísticamente significativas entre pre-test y post-test para la tutoría fija, $t(104)=-12.86, p<.01$, y también para el caso de la tutoría recíproca, $z=-2.38 ; p<.05$.

Por lo tanto, en comprensión y fluidez lectora los avances son estadísticamente significativos, independiente del tipo de tutoría asumida por los alumnos. A pesar de la prudencia con que se deben interpretar los resultados, debido a que en el caso de la tutoría recíproca el número total de implicados no superaba los 30 sujetos, los progresos podrían deberse a que en el caso de la tutoría fija, el tutor pone en marcha mecanismos de mediación para facilitar el aprendizaje de su tutorado, lo cual le permite también reconstruir y modificar sus propios esquemas cognitivos. En el caso de la tutoría recíproca, la oportunidad de mediación se otorga de igual manera a ambos miembros de la pareja para poner en marcha estrategias, enfrentar conflictos cognitivos, reestructurando sus esquemas cognitivos, generando o modificando los conocimientos que ya poseían. En resumen, poniendo en marcha una serie de mecanismos que facilitarían la construcción de conocimiento, tanto de ellos mismos como de sus compañeros de manera equitativa, al ir rotando los roles. 
Respecto a los resultados obtenidos por los alumnos/as, en relación a los roles asumidos (en la tutoría fija), se evidencian en la siguiente Tabla 3.

\section{TABLA 3}

Resultados de las variables comprensión y fluidez lectora en función del rol asumido por Tutores y tutorados.

\begin{tabular}{|l|l|l|l|l|l|l|l|l|l|}
\hline Variable & Rol & $\mathbf{N}$ & $\mathbf{M}$ Pre-test & SD & M Post-test & SD & $\mathbf{t}$ & $\mathbf{p}$ & $\mathbf{d}$ \\
\hline CL & Tutor & 54 & 64.91 & 15.40 & 74.85 & 13.89 & -7.59 & .00 & .68 \\
\hline \multirow{2}{*}{ FL } & Tutorado & 51 & 43.83 & 16.37 & 54.96 & 18.61 & -6.88 & .00 & .64 \\
\hline & Tutor & 54 & 68.42 & 18.09 & 83.40 & 17.04 & -7.87 & .00 & .85 \\
\hline
\end{tabular}

Los resultados alcanzados por los alumnos/as que asumieron el rol de Tutores muestran diferencias estadísticamente significativas entre la medición inicial y final, $t(53)=-7.59, p<.01$. En el caso de los tutorados también existen diferencias significativas tras finalizar el programa, $t(50)=-6.88, p<.01$.

Con el fin de profundizar en las diferencias observadas, se ha procedido a calcular el tamaño del efecto, constatándose en ambos casos que estos son medianos (Tutores $d=.68$; tutorados $d=.64$ ). Podemos señalar entonces que los alumnos/as, independientemente del rol asumido, muestran avances significativos en comprensión lectora y la tutoría tuvo un impacto relevante, indistintamente de los roles ejecutados.

En relación a la variable fluidez lectora, se puede apreciar que los estudiantes que asumieron el rol de Tutores alcanzaron avances estadísticamente significativos, con un tamaño del efecto grande, $t(53)=-7.87, p<.01, d=.85$. Respecto a los tutorados, también se evidenciaron diferencias estadísticamente significativas en relación a sus medidas iniciales y finales, y con un tamaño del efecto grande por sobre $1, t(50)=-$ $10.70, p<.01, d=1.15$.

Por lo tanto, independientemente del rol asumido por los alumnos, el programa ha demostrado ser eficaz para el mejoramiento de la fluidez lectora. 


\title{
3. Percepciones del alumnado y profesorado sobre la tutoría entre iguales
}

Las oportunidades de aprendizaje entregadas por el programa para tutores y tutorados permitieron que, aún partiendo de niveles iniciales de comprensión y fluidez lectora lógicamente diferentes (más altos para los alumnos tutores), todos los alumnos, independientemente del rol, mejoraran su nivel final. Lo que significa que llegan a niveles diferentes, pero alcanzando en todos los casos resultados estadísticamente significativos.

Al ser consultados los alumnos Tutores, sobre si realmente creían que se aprendía enseñando, el $97.8 \%$ están de acuerdo con la afirmación, argumentando que:

"se enseña y se aprende cosas que no sabemos" (T9); "porque enseñas a tu tutorado y además aprenden cosas nuevas los dos" (T12); "porque se aprende uno del otro" (T17); "porque aprendes cosas de tu tutorado y de las cosas que lees" (T23); "a la vez que enseño a mi tutorado yo también aprendo" (T41); "porque en algún texto, cuando le estás explicando de que va, el tutorado te dice las cosas que sabe sobre el tema" (T49). Así, los tutores son conscientes de que se aprende porque se conoce nueva información de los textos, gracias a los conocimientos que poseen los tutorados; también cuando se ponen en marcha determinadas estrategias de mediación; la labor de enseñar puede ser aprendida; y, el aprendizaje alcanzado es mutuo para tutor y tutorado.

Por otro lado, al ser consultados los tutorados si creían haber aprendido de sus compañeros, el $8.8 \%$ se muestran de acuerdo con la afirmación, argumentando así que:

\begin{abstract}
"si alguna cosa tú no sabes, el tutor te la puede decir" (t78); "porque los tutores aprenden de los tutorados y los tutorados de sus tutores" (t82); "porque te suelen enseñar cosas nuevas y al ser personas más cercanas les entiendes mejor" (t89); "es como un profesor, me hace preguntas y yo respondo" (t100); "nos ayudan en todo" (t103); "se aprende mucho con los compañeros" (t115); "me enseñan cosas porque se prepara la lectura en casa" (t122).
\end{abstract}

En este sentido, los tutorados destacan la adquisición de nuevos conocimientos; el apoyo de sus tutores para solucionar dificultades de comprensión; el dominio del contenido del texto que posee el tutor, gracias a la preparación previa, lo cual facilita la construcción de aprendizajes de los tutorados. Además, se destaca la imagen de profesor que los tutorados le atribuyen a sus tutores, pero haciendo notar un elemento fundamental: la cercanía y proximidad con su enseñante lo cual favorecería 
el proceso de construcción de la comprensión lectora. En general, ha sido recurrente el reconocer la ayuda prestada en todo momento por sus tutores, como un soporte y guía constante durante el desarrollo de las actividades.

En relación al análisis de los testimonios de los profesores, estos respaldaron la tutoría entre iguales como un método que permite aprender y mejorar a los alumnos con mayores dificultades, no solo en la competencia lectora, sino también en la competencia social. Destacan el hecho de que tener un mediador próximo a su contexto, nivel, intereses... potencia la relación de aprendizaje que se pueda establecer y los conocimientos que se puedan alcanzar. Todo ello se sintetiza en el siguiente extracto:

"para los alumnos con dificultades me parece muy positivo que sea un compañero quien le ofrezca las ayudas y quien le rectifique, ya que siempre es un ser más cercano que el profesor con un mayor grado de confianza. En general, pienso que es un programa muy válido para todos incluidos los niños con dificultades" (P6).

Finalmente, se destaca la importancia de la preparación previa de las hojas por parte de los tutores, constatándose como un factor fundamental a la hora de asegurar el éxito de la sesión y sobre todo la construcción de los aprendizajes.

\section{CONCLUSIONES}

Las redes de cooperación establecidas en el aula entre los alumnos, y organizadas debidamente por sus profesores, repercutieron positivamente en el progreso de los índices de comprensión y fluidez lectora de los 127 alumnos participantes del programa, en contraste de lo evidenciado con el grupo de comparación. Si bien en este artículo no presentamos datos de la interacción en el seno de las parejas, los resultados cualitativos parecen indicar que la responsabilidad de esos avances se basan fundamentalmente en las ayudas andamiadas puestas en marcha por el alumno tutor. Ellas han permitido ir regulando y monitorizando el proceso cognitivo llevado a cabo por parte de ambos alumnos/as, promoviendo de esta manera la enseñanza y aprendizaje implícita de las estrategias metacognitivas, que Van Keer y Verhaeghe (2005) destaca como imprescindibles en este proceso de construcción mutua, además de facilitar el avance en la zona de desarrollo próximo del tutorado, siendo este el protagonista y constructor de sus aprendizajes. 
Respecto a la variable fluidez lectora, la gestión del aula por medio de de la tutoría entre iguales también permitió evidenciar progresos y un impacto significativo en la evolución de este componente. En este sentido parece consecuente atribuir dichas ganancias a las prácticas de lectura en voz alta en parejas, retroalimentación mutua del proceso, modelaje de parte del tutor y construcción conjunta de la comprensión del texto.

El avance de estos alumnos/as en particular, y en esta experiencia implementada en el seno de escuelas aragonesas, está en consonancia también con otros programas en el área de la lectura basados en la tutoría entre iguales, con un formato estructurado de interacción, que conjugado con las actuaciones habituales de los docentes en el aula, promovieron también el mejoramiento de la competencia lectora, como en el caso de los programas Paired Reading (Topping, 1989; Topping y Whiteley, 1990), Read On (Topping y Hogan, 1999), Reading Together (Hattie, 2006) y Buddy Reading (Shegar, 2009).

El profesorado, tras su participación, valora el aprendizaje cooperativo, en este caso la tutoría entre iguales, como una estrategia útil e innovadora para alcanzar una comprensión lectora profunda, para atender la diversidad del aula -utilizando en positivo las diferencias de conocimiento que tienen los alumnos- y en especial a los alumnos que poseen dificultades de aprendizaje, y destacando el valor de la cooperación, como una competencia valiosa y necesaria para convivir en sociedad. 


\section{REFERENCIAS}

Blanch, S., Duran, D., Valdebenito, V. y Flores, M. (2013). The effects and characteristics of family involvement on a peer tutoring programme to improve the reading comprehension competence. European Journal of Psychology of Education, 28, 101-119.

Borisov, C. y Reid, G. (2010). Students with intellectual disabilities acting as tutors: an interpretative phenomenological analysis. European Journal of Special Needs Education, 25(3), 295-309.

Byrd, D. (1990). Peer tutoring with the learning disabled: A critical review. Journal of Educational Research, 84(2), 115-118.

Català, G., Comes, G. y Renom, J. (2001). Evaluación de la comprensión lectora. Barcelona: Graó.

Cohen, P., Kulik, J. y Kulik, C. (1982). Educational outcomes of tutoring: A metaanalysis of findings. American Educational Research Journal, 19(2), 237-248.

Cortese, C. (2005). Learning through teaching. Management Learning, 36(1), 87-15.

Delors, J. et al. (1996). La educación encierra un tesoro. Madrid: Santillana.

Díaz-Aguado, M. J. (2004). Educación intercultural y cooperación. Una nueva interacción educativa para un mundo que también es diferente. Educatio Siglo $X X I, 22,59-89$.

Duran, D. (2011). Aprender enseñando: un paradigma emergente. Herramientas, 110, 4-12.

Duran, D., Blanch, S., Corcelles, M., Flores, M., Oller, M., Utset, M. y Valdebenito, V. (2011). Leemos en Pareja. Tutoría entre Iguales para la Competencia Lectora. Barcelona: Horsori.

Duran, D., Blanch. S., Dekhinet, R. y Topping, K. J. (2010). Una experiencia de tutoría 
entre iguales virtual para el aprendizaje del castellano y el inglés. Textos de Didáctica de la Lengua y la Literatura, 53, 89-101.

Duran, D., Blanch. S., Thurston. A. y Topping, K. (2010). Tutoría entre iguales recíproca y virtual para la mejora de habilidades lingüísticas en español e inglés. Infancia y Aprendizaje, 33 (2), 209-222.

Duran, D. y Huerta, V. (2008). Una experiencia de tutoría entre iguales en la Universidad mexicana de Oaxaca. Revista Iberoamericana de Educación, 48, 1-15.

Duran, D. y Sánchez, G. (2012). Ritmos en Dos: una experiencia basada en la Tutoría entre iguales para la mejora de la fluidez y comprensión de lectura rítmica musical. Eufonía. Didáctica de la Música, 56, 99-106.

Duran, D. y Vidal, V. (2004). Tutoría entre iguales: de la teoría a la práctica. Un método de aprendizaje cooperativo para la diversidad en secundaria. Barcelona: Graó.

Fernández, M. (2007). Redes para la innovación educativa. Cuadernos de Pedagogía, 374, 26-30.

Flick, U. (2004). Introducción a la investigación cualitativa. Madrid: Ediciones Morata. García Vidal, J. y González, D. (1999). Batería Psicopedagógica Evalúa 4. Madrid: EOS.

García Vidal, J, Manjón, D. y Ortiz, B. (2006). Batería Psicopedagógica Evalúa 5. Madrid: EOS.

Garzón, M. C., Jiménez, M. E. y Seda, I. (2008). El teatro de lectores para mejorar la fluidez lectora en niños de segundo grado. Lectura y Vida, 1, 33-44.

Gil, J. (2011). Hábitos lectores y competencias básicas en el alumnado de educación secundaria obligatoria. Educación XX1, 14(1), 117-134.

Ginsburg-Block, M. D., Rohrbeck, C. A. y Fantuzzo, J. W. (2006). A meta-analytic review of social, self-concept, and behavioral outcomes of peer-assisted learning. Journal of Educational Psychology, 98(4), 732-749. 
Hattie, J. (2006). Cross-age tutoring and the reading together program. Studies in educational evolution, 32(2), 100-124.

Janssen, J., Kischner, F., Erkens, G., Kischner, P. y Pass, F. (2010). Making the black box of collaborative learning transparent: Combining process-oriented and cognitive load approaches. Educational Psychology Review, 22, 139-154. DOI 10.1007/s10648-010-9131-x

Johnson, D.W. y Johnson, R. (2009). An Educational Psychology Success Story: Social Interdependence Theory and Cooperative Learning. Educational Researcher, 38(5), 365- 379. DOI: 10.3102/0013189X09339057.

Johnson, B., y Onwuegbuzie, A. (2004). Mixed methods research: A research paradigm whose time has come. Educational researcher, 33(7), 14-26.

McMaster, K., Fuchs, D. y Fuchs, L. (2006). Research on peer-assisted learning strategies: The promise and limitation of peer-mediated instruction. Reading and Research Quarterly, 22, 5-25.

Mayoral, P. y Corcelles, M. (2008). Una experiència de treball cooperatiu per aprendre matemàtiques a primària: la maleta de geometria. Suports, 1, 13-20.

Mineduc (s/f). Planes de mejoramiento lector. Recuperado el 25 de septiembre de 2012 desde http://www.planesdemejoramiento.cl/m_anexos/Anexo\%20IV.pdf

Moliner, L., Flores, M. y Duran, D. (2011). Efectos sobre la mejora de las competencias lingüísticas y de autoimagen lectora a través de un programa de tutoría entre iguales. Revista de Investigación en Educación, 9(2), 209-222.

Monereo, C., Castelló, M., Bassols, M. y Miquel, E. (2000). Instantáneas. Proyectos para atender la diversidad educativa. Madrid: Celeste.

Moreno, J. (2007). Torneo combinado. Una experiencia de aprendizaje cooperativo en tercer curso de ESO. Aula de Innovación Educativa, 158, 41-46.

Mosca, A. y Santiviago, C. (2012). Fundamentos conceptuales de las tutorías entre pares. 
La experiencia de la Universidad de la República. Montevideo: Universidad de la República.

Nichols, W., Rupley, W. y Rasinski, T. (2009). Fluency in learning to read for meaning: going beyond repeated readings. Literacy Research and Instruction, 48(1), 1-13.

Organización para la Cooperación y el Desarrollo Económicos (2009). Assessment Framework. Key competencies in reading, mathematics and science. París: OECD. Recuperado el 11 de Octubre de 2012 desde http://www.oecd.org/ dataoecd/11/40/44455820.pdf

Oakhill, J. y Cain, K. (2007). Issues of causality in children's reading comprehension. En D. McNamara (Ed.), Reading comprehension strategies: Theories, interventions, and technologies (pp. 47-42). New York: Erlbaum.

Okilwa, N. y Shelby, L. (2010). The Effects of Peer Tutoring on Academic Performance of Students with Disabilities in Grades 6 Through 12: A Synthesis of the Literature. Remedial and Special Education, 31(6), 450-463. DOI: 10.1177/0741932509355991.

Pearson, P. D. y Hamm, D. N. (2005). The assessment of reading comprehension: A review of practices-paste, present, and future. En S. G. Paris y S.A. Stahl (Eds.), Children's Reading comprehension and assessment (pp. 13 -69). Mahwah, NJ: Erlbaum.

Robinson, D. R., Schofield, J. y Steers-Wentzell, K. L. (2005). Peer and CrossAge Tutoring in Math: Outcomes and Their Design Implications. Educational Psychology Review, 17(4), 327-362.

Rohrbeck, C., Ginsburg-Block, M., Fantuzzo, J. y Miller, T. (2003). Peer-assisted learning interventions with elementary school students: A meta-analytic review. Journal of Educational Psychology, 95(2), 240-257.

Rychen, D.S. y Salganik, L.H. (2003) (Eds.). Key competencies for a successful life and a well-functioning society. Göttingen: Hogrefe \& Huber. 
Shegar, Ch. (2009). Buddy Reading in a Singaporean primary School: Implications for training and research. RELC Journal, 40(2), 133-148.

Spencer, V. G. (2006). Peer Tutoring and Students with Emotional or Behavioral Disorders: A Review of the Literature. Behavioral Disorders, 31(2), 204-222.

Strijbos, J.W. y Fischer, F. (2007). Methodological challenges for collaborative learning research. Learning and Instruction, 17, 389-393.

Thurston, A., Van de Keere, K., Topping, K, J., Kosack, W., Gatt, S., Marchal, J., Mestdagh, N., Schmeinck, D., Sidor, W. y Donnert, K. (2007). Aprendizaje entre iguales en Ciencias Naturales de Educación Primaria: Perspectivas teóricas y sus implicaciones para la práctica en el aula. Revista Electrónica de Investigación Psicoeducativa, 5 (3), 477-496. Recuperado el 2 de octubre de 2012 desde http://grupsderecerca.uab.cat/grai/es/content/art\%C3\%ADculos

Topping, K. J (1987). Peer tutored paired reading: outcome data for ten projects. Educational Psychology, 7(2), 45-133.

Topping, K. J. (1988). The peer tutoring handbook: Promoting cooperative learning. London: Croom Helm.

Topping, K. J. (1989). Lectura conjunta: Una poderosa técnica al servicio de los padres. Comunicación. Lenguaje y Educación, 3(4), 143-151.

Topping, K. J. (2005). Trends in peer learning. Educational Psychology, 25(6), 631645.

Topping, K.J. y Hogan, J. (1999). Read On: Paired reading and Thinking video resource pack. Londres: BP Educational Services.

Topping, K. J., Miller, D., Thurston, A., McGavock, K. y Conlin, N. (2011). Peer tutoring in reading in Scotland: thinking big. Literacy, 45(1), 3-9.

Topping, K.J. y Whiteley, M. (1990). Participant Evaluation of Parent-Tutored and Peer-Tutored projects in Reading. Educational Research, 32(1), 14-32. 
Utset, M. y Duran, D. (En prensa). Red Leemos en pareja: un modelo de formación docente, basado en el aprendizaje entre iguales, para la sostenibilidad de la innovación educativa. Cultura y Educación.

Valdebenito, V. y Duran, D. (2010). Implicación familiar en un programa de tutoría entre iguales para la mejora de la comprensión y la velocidad lectora. En J.J. Gázques y M.C. Pérez, Investigación en Convivencia Familiar. Variables relacionadas (pp. 433438). Granada: GEU.

Van Keer, H., y Verhaeghe, J. P. (2005). Effects of explicit reading strategies instruction and peer tutoring in second and fifth graders' reading comprehension and selfefficacy perceptions. The Journal of Experimental Education, 73, 291-329.

Vidal, J., Duran, D. y Vilar, M. (2010). Aprendizaje musical con métodos de aprendizaje cooperativo. Cultura y Educación, 22(3), 363-378.

Wheldall, K. y Colmar, S. (1990). Peer tutoring in low-progress readers using pause, prompt and praise. En H. Foot, M. Morgan y R. Shute (Eds.). Children helping children (pp. 117-134). Chichester: John Willey and Sons. 